
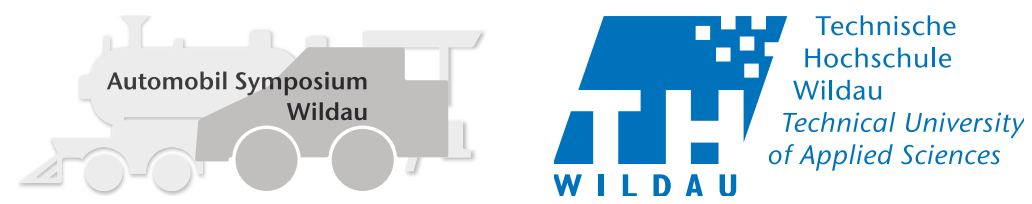

\title{
Kritische »Change Readiness« der Automobilindustrie angesichts der neuen IT Eco-Systeme: Wie kann die digitale Transformation dennoch gelingen?
}

Hagen Ringshausen

doi: 10.15771/ASW_2016_1

»This digital era will dwarf what's occurred in the information era and the value of the Internet today. As leaders, if you don't transform and use this technology differently - if you don't reinvent yourself, change your organization structure; if you don't talk about speed of innovation - you're going to get disrupted. And it'll be a brutal disruption, where the majority of companies will not exist in a meaningful way 10 to 15 years from now«. John Chambers, CEO, Cisco Systems, March 2016 (Chambers \& Kirkland 2016).

\section{Zusammenfassung}

»Embracing Disruption«, »Digital Trust Building« sowie "Liquid Workforces« bezeichnen nur drei der wesentlichen globalen IT Trends, die auch die Automobilindustrie vor allem in Deutschland bereits heute vor enorme Anstrengungen stellt, wobei viele Unternehmen sich noch immer in gefährlicher Weise abwartend verhalten. Nach Einschätzung des Autors werden bis zu $50 \%$ der Firmen in der deutschen Automobilbranche die nötigen strukturellen Transformationen nicht rechtzeitig bzw. nicht ausreichend nachhaltig organisieren, so dass innerhalb der nächsten 10 Jahre mit einer völlig veränderten Branchenstruktur zu rechnen sein wird.

Der Beitrag nimmt fokussiert eine Binnenperspektive innerhalb der Unternehmensorganisationen und ihrer Transformationsbedingungen ein. Diese reflexiven Betrachtungen treffen strukturell sowohl auf KMUs als auch auf die Konzerne der OEMs zu: Anhand kurzer Schlaglichter werden die Hauptpunkte erforderlicher Transformationen innerhalb der Unternehmensorganisation aufgezeigt und konsequente Handlungsableitungen im Sinne einer gelingenden digitalen Transformation skizziert.

1. „Change Readiness Concept« - ein geeignetes Frühwarnsystem und kaum beachtetes Phänomen zur Einschätzung unternehmerischer Zukunftsfähigkeit

1.1 Zum "Status Quo« einer Vorzeigebranche: Organisationale Unbeweglichkeit am Anfang der neuen Digitalisierungswelle

Die Automobilindustrie im Land der Autobauer ist nicht nur seit Jahrzehnten eine, wenn nicht »die« Vorzeigebranche Deutschlands, sondern sie ist vor allem auch eines, nämlich: innerhalb der einzelnen OEM-Organisationen organisatorisch zu hochkomplexen, vergleichsweise »unbeweglichen Organisationsungetümen« angewachsen.

Dieser Umstand erklärt sich bei Weitem nicht alleine über die Konzerngröße als »global player«, sondern vielmehr aufgrund der enormen Arbeitsteilung und Regelungstiefe, wie sie sich in bürokratischen Formen wie Konzernrichtlinien oder aktuell beispielsweise in dezidierten Compliance Vorschriften niederschlägt. Hinzu kommt der wirtschaftlich ungebrochene Erfolg der letzten Jahrzehnte, der die Branche trotz steigendem Wettbewerbsdruck, nicht gerade auf »Change Management Expertise« getrimmt hat: Schlussendlich stehen wir vor einem wiederkehrenden Organisationsphänomen zu Beginn eines neuen digitalen Zeitalters, nur dass das Wiederauftreten jetzt »zur maximalen Unzeit« geschieht, wie auch Cisco System CEO John Chambers es illustrativ darstellt (s. o. Zitat): Standardisierung trifft unternehmerische Freiheit.

Bei genauerer Betrachtung repräsentiert die Führungsund Unternehmenskultur hierbei vielleicht den größten Hemmschuh, wenn es aktuell darum geht, sich als OEM angesichts der neuesten Entwicklungen bei Apple und Google rund um das eCar und anderer gesellschaftlicher Werteverschiebungen (z. B. mieten statt besitzen) flexibler aufzustellen. Die traditionelle Kultur ist strukturell deutlich mehr auf die Reproduktion alter Strukturen und Prozesse ausgelegt als auf kontinulierliche Erneuerung und Agilität:

Bei der Analyse von push-and-pull-Effekten müssen wir auf der einen Seite unterscheiden zwischen der Bewältigung neuer organisationsstruktureller Herausforderungen, wie sie als Ergebnis und Ausdruck der gesamtgesellschaftlichen Digitalisierung in der Frage »Wie baut man einen Automobilbauer von innen so um, dass er der Digitalisierung in Bezug auf seine Hauptprodukte Personen- und Lastkraftwagen wettbewerbsfähig möglichst optimal aufgestellt ist?« (Man könnte den planerischen Flexibilitätsdruck gedankenspielerisch sicherlich dadurch noch weiter erhöhen, dass man exemplarisch das Internet der Dinge noch hinzudenkt, das neben Fragen von 
Safety und Security kommunizierender Fahrzeuge (vgl. Beitrag von Tilo Schneider und Roland Melster) auch Fragen zukünftiger, produktbezogener Innovationsraten aufwirft und die deutsche Dauerschwäche «time-tomarket« auf den Plan ruft).

Auf der anderen Seite müssen wir vor allem die kulturellen, innenpolitischen Beharrungskräfte erkennen, die dem marktgetriebenen, dringend erforderlichen Umbau der internen Organisationsstrukturen entgegenstehen. Es werden zunehmend Stimmen lauter, die angesichts der sich beschleunigenden Digitalisierung einen neuen Typus von Managern fordern: Begeisterungsfähige Leader, interdisziplinär denkend, multikulturelle Teams führend, Change-Management kompetent und hochintegrativ: Die Eckpunkte dieses Führungsprofils setzen wir nicht unweigerlich gleich mit den heutigen, tonangebenden Entwicklungsingenieuren bei Mercedes, BMW oder Audi.

An diese kritische Bestandsaufnahme schließt sich nun das Konzept von Change Readiness oder Change Agility treffend an.

\subsection{Was ist das "Change Readiness Concept« zur} Evaluation organisationaler Agilität?

Im Kern geht es hierbei um das Maß an Vertrauen der Organisation in der Gesamtheit aller internen und externen Stakeholder zur Erfüllung der angestrebten, unternehmerischen Wirtschaftsziele.

Das wirtschaftspsychologische Konzept beruht im Wesentlichen auf drei Elementen:

1. den organisationalen Veränderungskapazitäten/ bzw. der Change Management Expertise,
2. dem Commitment der Führungskräfte und der Mitarbeiter zu den strategischen Unternehmenszielen sowie

3. der Unternehmenskultur selbst.

Was das Konzept in seiner Anwendung komplex macht, ist die Vielfältigkeit seiner Datenbasis bzw. seine sehr heterogenen Datenniveaus: Subjektive Wahrnehmungen und Einschätzungen beispielsweise von Vorständen, Aufsichtsräten oder Firmeneignern, die Arbeitgeberattraktivität in regionalen wie überregionalen Recruitingmärkten ebenso wie vertriebsbezogene, kaufmännische Marktpotenzialanalysen kommen hier komplementär zusammen.

Entscheidender als die letzte mathematische Präzision im Ergebnis ist die Früherkennung etwaiger Transformationsrisiken vor Beginn einer geplanten Reorganisation, also von einer »ausreichenden « bis zu einer "guten/sicheren Change Readiness « oder eben einer »kritischen bis ungenügenden Change Readiness«. Letztere würde den Erfolg der Transformation in Frage stellen. Ein eindrucksvolles Beipiel für eine äußerst geringe Change Readiness haben wir zuletzt in der gescheiterten Fusion zwischen Daimler und Chrysler erleben können: Nur wenn in allen drei Bereichen ein hoher Reifegrad erreicht wird, kann auch davon ausgegangen werden, dass die digitale Transformation innerhalb kurzer Zeit erfolgreich gelingen kann.

Zur Ermittlung der gesamtbetrieblichen Veränderungsbereitschaft und -kompetenz werden üblicherweise 1-2 mal jährlich entsprechende Checklisten und Fragebögen eingesetzt.

Wie es wiederum John Chambers von Cisco System ausdrückt, sollte der Fokus der digitalen Organisations-

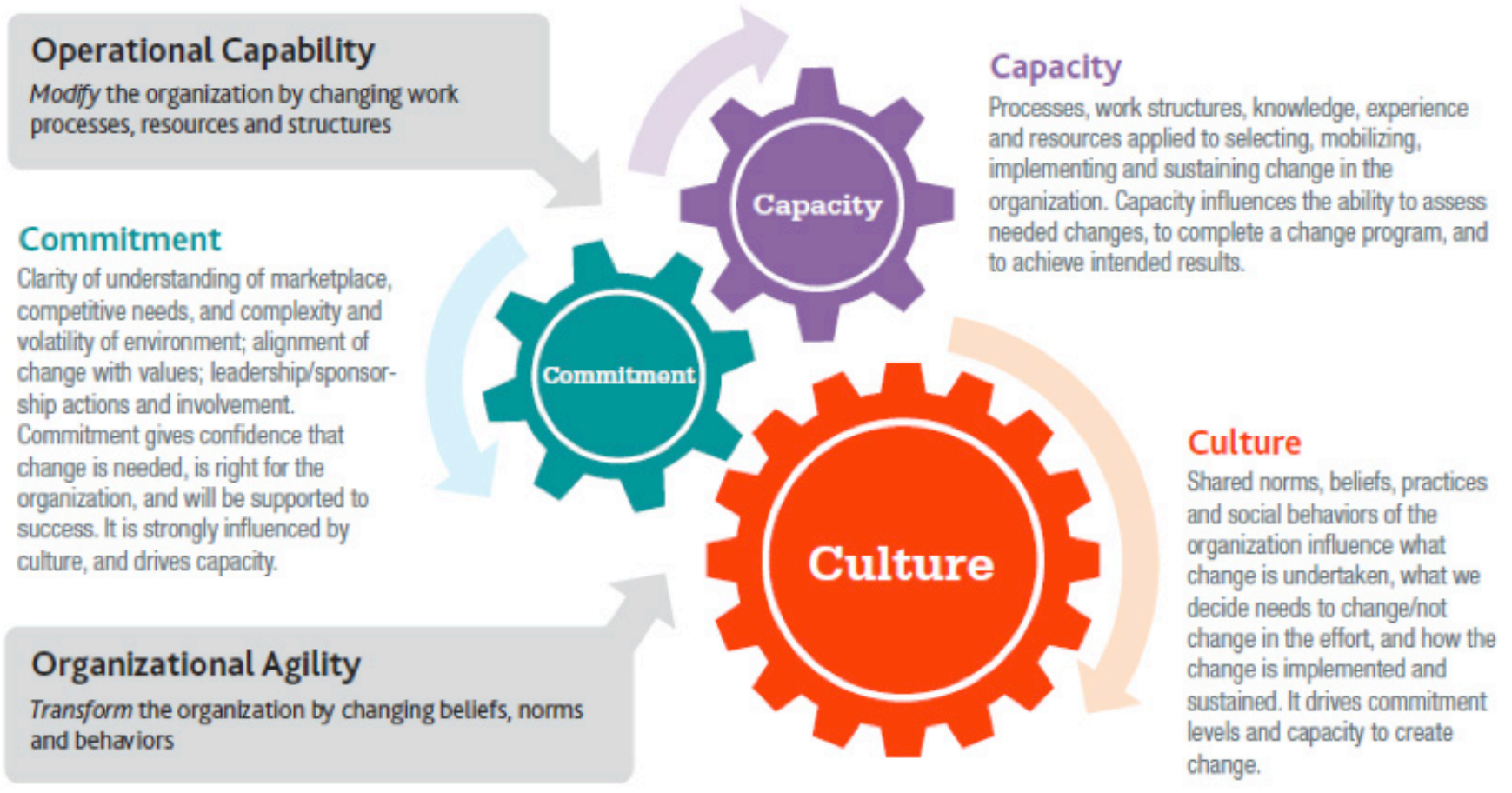

Abb. 1 Drivers of Organizational Change Agility (Combe 2014b: 7) 
entwicklung zu Beginn der neuen Digitalisierungsära stringent auf dem Ziel »cultural simplicity« gesetzt werden, um Effizienz, Produktivität und Geschwindigkeit für innovative Produkt- und Serviceentwicklungen wettbewerbswirksam nachhaltig zu steigern. Dieser Punkt soll am Ende des Artikels im Zusammenhang mit end-toend-operating models nochmals aufgegriffen werden.

\section{Die organisationalen Hauptherausforderungen der digitalen Transformation}

Die folgenden Kurzbeschreibungen von drei weitreichenden IT Trends, die sehr zentrale Herausforderungen für die Automobilbranche darstellen, sind einem Stratgiepapier von Accenture entnommen. Accenture versucht mit dieser »Technology Vision 2016« die stärksten Treiber der Digitalisierung zu identifizieren und gleichzeitig erste Handlungsempfehlungen auszusprechen, wie Unternehmen sich wirtschaftlich erfolgreich dafür aufstellen können.

\section{1 »Embracing Disruption«}

Durch die zunehmende Digitalisierung werden sich klassische Branchengrenzen und Industriezweige weiter auflösen. Derzeit erleben wir dieses Phänomen eindrucksvoll an der Frage, wie die IT Infrastruktur im Zuge des autonomen Fahrens bzw. des eCars dazu führen wird, dass beide Branchen: Automobilindustrie und luK-Industrie zumindest in Teilen synergetisch verschmelzen könnten. Plattformgestützte Geschäftsmodelle transformieren die heute bekannten Industriegrenzen zunehmend zu neuen Ecosystemen, ohne dass zwischen den marktbeherrschenden Brands bereits strategische Allianzen im nennenswerten Umfang sichtbar wären.

Eine große, beinahe »tragische« Gefahr besteht offensichtlich darin, dass gerade Großunternehmen und Konzerne wie OEMs sich zulange mit organisatorischen Fragen der Repositionierung ihrer IT Funktion im eigenen Unternehmen beschäftigen, bevor der digitale Nutzen bei alten und neuen Kunden ankommen kann. Diese Selbstbeschäftigung erschwert auch die Herausbildung neuer smarter Geschäftsmodelle zwischen den marktführenden Playern. Zwar geben laut einer aktuellen Studie von Accenture zur Digitalisierung der Wirtschaft $81 \%$ der über 3.100 weltweit befragten Unternehmen an, dass sie digitale Geschäftsnetzwerke innerhalb ihrer Branche aufmerksam beobachten; damit verhalten sich die meisten von ihnen zu passiv und wenig proaktiv. In der Zwischenzeit - so kann zumindest vermutet werden - sind kapitalstarke Firmen wie Apple, Google oder Tesla in der Lage, entlang hochinnovativer Produktforschungen genau die interdisziplinären Experten zu recrutieren und an sich zu binden, um weltweit die Technologieführerschaft des digitalisierten Automobils sukzessive zu übernehmen.

Intere funktions- und extern branchenübergreifende $\mathrm{F}+\mathrm{E}-$ Kooperationen und Allianzen sind derzeit sicherlich eine enorm wichtige strategische Investionsleistung, um den Anschluß technologisch nicht zu verlieren und um den kostenintensiven Organisationsumbau der »old economy« zum »digital enterprise« eventuell bewältigen zu können. »New IT« heißt vor allem eines: Geschwindigkeit und Agilität der Organisation(smitglieder).

Anstatt die üblichen wirtschaftlichen und zeitlichen Reibungsverluste für Reorganisationsphasen weiterhin in Kauf zu nehmen, müssen »digital winner« neben dem höchsten Technologiestandard eben auch eines mitbringen, das erst neuerdings in den Fokus der Aufmerksamkeit geraten ist: nämlich die uneingeschränkte Bereitschaft der Belegschaft zur kontinuierlichen Veränderung ohne individuelle Ängste vor Performance- Einbußen, Verlust von Handlungsroutinen oder Sorgen angesichts einer zunehmend volatilen, unklaren Zukunft: Die Change Management Expertise wird für Organisationen wie für Individuen zur neuen »zivilisatorischen Schlüsselkompetenz« im globalen Dorf.

\section{2 »Digital Trust Building«}

Die Möglichkeiten der branchenübergreifenden Zusammenarbeit werden sich aufgrund neuer platformgestützter Kooperationsoptionen schneller ausbreiten als sich überlicherweise zwischenmenschliches Vertrauen zwischen zwei Individuen im Sinne eines belastbaren, empathiegeprägten, produktiven Arbeits- und Beziehungssettings herstellen lässt. Insofern ist es höchst wichtig, dass Unternehmer genau hier die unternehmerischen Vorstöße ihrer Mitarbeiter und Führungskräfte zu neuen Koopertionsformen - häufig über social media organisiert - fördern und aktiv unterstützen. Dies kann zum einen durch die verstärkte Bereitstellung eines entsprechenden innovationsgebundenen $\mathrm{F}+\mathrm{E}$-Budgets geschehen. Zukünftige Innovationszyklen werden tendenziell immer kürzer, insofern müssen auch immer kurzfristiger $\mathrm{F}+\mathrm{E}-$ Investitionen geplant und realisiert werden.

Der andere essentiell wichtige Erfolgsfaktor ist das Agieren unter den höchsten ethischen Standards zur gemeinsamen Nutzung von »big data« und kollektiven IT-Infrastrukturen, die damit auch in digitalen Zeiten ein Höchstmaß an virtueller Verbundenheit und einer neuen Form der globalen Wertegemeinschaft ermöglichen könnten.

\section{3 »Liquid Workforces« - People first}

Auch wenn in den vielfältigen, aktuell laufenden Diskussionen über »Industrie 4.0«, »Internet der Dinge« und »smart world« etc. die Technologie vordergründig thematisiert wird, so sind es doch letzten Endes stets die Menschen, die die digitale Transformation wollen, planen, umsetzen und kontrollieren müssen.

»Digital Trust Building « und »Liquid Workforces« werden gerade für das häufig traditionell geprägte Führungsverständnis in der old economy eine außergewöhnliche kulturelle Transformation bedeuten. Angesichts des unge- 
brochenen Fach- und Führungskräftemangels, der nach wie vor auch in der Automobilindustrie existiert, ergänzt durch das abnehmende Loyalitäts- und Bindungsverhalten von VertreterInnen der »Generation $Y$ und $Z$ « gegenüber ihrem Arbeitgeber, wird gerade das strategisch relevante Personal immer mehr zur »Achillesferse« vieler Unternehmen, die sich dadurch ihr Innovationspotential häufig nicht mehr rechtzeitig bzw. im nötigen Umfang einkaufen können. Daher muss heute zur Sicherung der Zukunftsfähigkeit mehr denn je gelten: People first! Dementsprechend benötigen die Marktführer von morgen hochattraktive, wettbewerbsdifferenzierende Unternehmenskulturen, die zunächst smarte Menschen anziehen bzw. binden können. Im zweiten Schritt müssen diese Menschen befähigt werden, mit intelligenter IT(Infrastruktur) Innovation und Veränderung zu gestalten: individuell, betriebsintern, organisationsweit, in Bezug auf Kunden, Lieferanden und Partnerfirmen sowie sämt- liche sonstige Stakeholder. Und schließlich sind sie es vor allem, die jungen, universitär ausgebildeten Menschen ohne praktische Berufserfahrung, die wesentlich die Change Readiness ihres Unternehmens und damit dessen Zukunftsfähigkeiten mitprägen werden: Reinventing the World again and again ...

3. Erste Ansätze zur Steigerung der Change Readiness und damit zur Bewältigung der digitalen Transformation

Wie gezeigt wurde, wird die neue digitale Transformation strukturelle Anforderungen an den organisationalen Wandel aller Unternehmen richten, die in dieser Nachhaltigkeit und Geschwindigkeit bislang nicht da gewesen sind.

McKinsey hat die beschriebene Ausgangsbasis ebenfalls erkannt und folgende Handlungsempfehlungen dage-

\section{An end-to-end operating model rests on three fundamentals.}

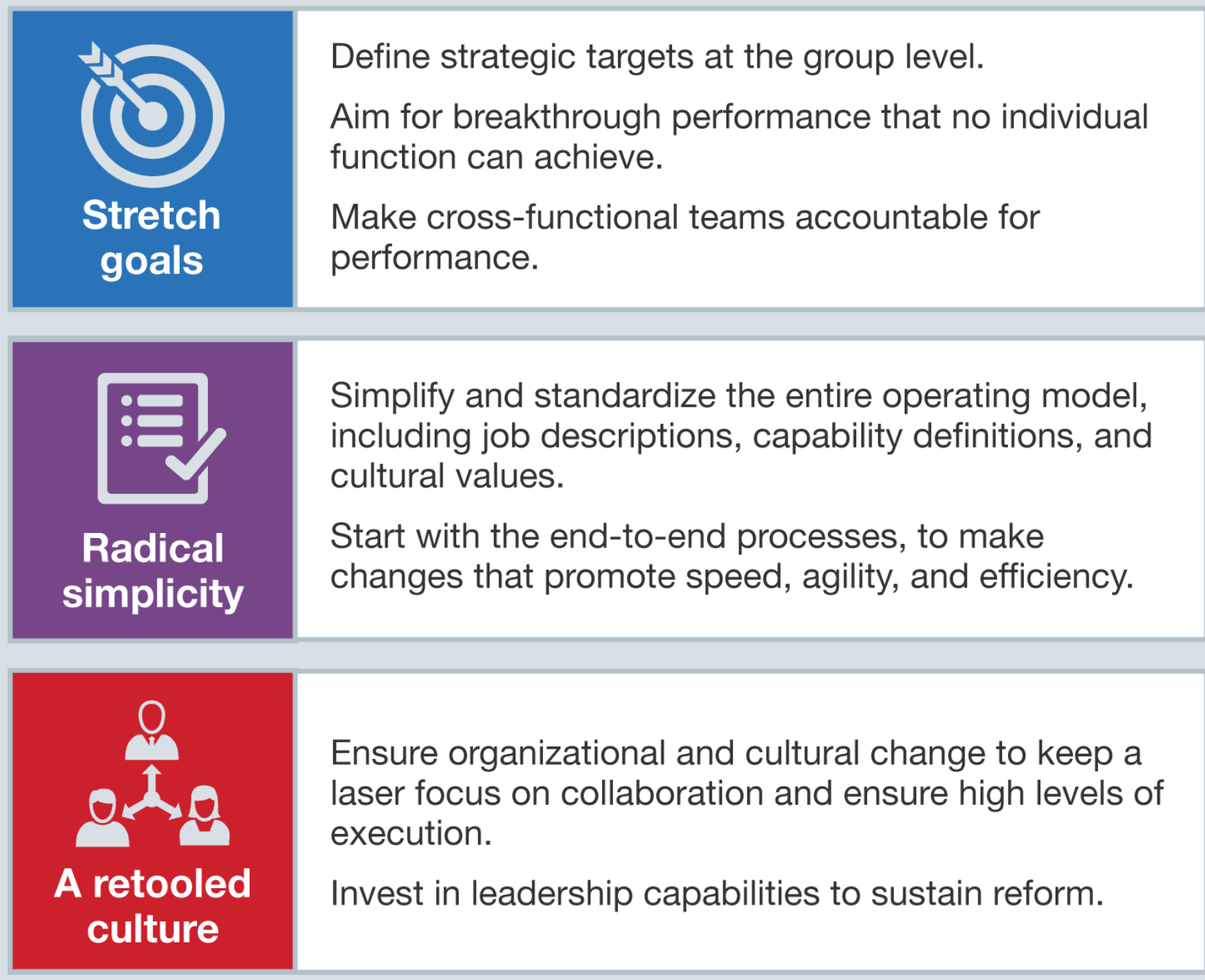

McKinsey\&Company

Abb. 2 (Schaubroeck et al. 2016) 
gen gestellt, die eine erste Orientierung zur Planung der organisationalen Transformation geben sollen (vgl. Abb. 2).

\subsection{Strech Goals/Share Visions}

Funktions- und bereichsübergreifende Wirtschaftsziele, die nur gemeinsam im Team erreicht werden können, fördern das Kommunikations- und Kooperationsverhalten im Unternehmen. Produktivitätsbezogene Synergien können dadurch entstehen und die Identifikation insbesondere von »high potentials« und »high performern« mit dem Unternehmen sollte damit ebenfalls signifikant gesteigert werden können.

\subsection{Radical Simplicity}

Geschäftsmodelle müssen radikal vereinfacht werden, um die nötige Agilität am Markt hervorbringen zu können. Geschwindigkeit, Effizienz und Flexibilität können nur in prozessual organisierten Unternehmen signifikant gesteigert werden. Prozesse müssen hierbei als vollständige Wertschöpfungsketten von allen beteiligten Akteuren auf allen Prozessstrecken verinnerlicht und verantwortungsbewusst gelebt werden. Das kann im Grunde nur gelingen, wenn die Freiheitsgrade des einzelnen Managers sich mehr und mehr den unternehmerischen Freiheitsgraden der operativen Geschäftsleitung annähern. Auch wenn Rückkopplungs- und Entscheidungsrituale top down nicht obsolet werden, so kann die erforderliche Agilität nur dann erreicht werden, wenn dezentral unternehmerische Entscheidungen grundsätzlich möglich und gewünscht sind, wie dies der Autor auch schon ausführlich an anderer Stelle dargelegt hat (vgl. Ringshausen 2000).

\subsection{A retooled culture}

Erfolgreiche Unternehmen müssen durch ihre kulturellen Rahmenbedingungen im wesentlichen zwei Dinge sicherstellen: eine hocheffiziente Kooperation aller Wertschöpfungsmitverantwortlichen an einem Prozess einerseits und eine rasche, markt- und kundengerechte Umsetzung andererseits. Führungskräften und Vorgesetzten als dezentralen Vor-Ort-Kulturverantwortlichen kommt dabei eine ganz besondere unternehmerische Mitverantwortung zu, um den permanenten Kooperations- und Innovationsgeist vorzuleben, anzuregen und kontinuierlich aufrechtzuerhalten. Hierfür werden selbst erfahrene Führungskräfte, lange Jahre geprägt durch die Vorzeichen der »old economy«, sicherlich noch zu sensibilisieren und zu trainieren sein ...

\section{Literatur}

Accenture (2016a) Accenture Technology Vision 2016. Executive Summary. www.accenture.com/technologyvision\#techvision2016. Accessed 28 Mar 2016
Accenture (2016b) Die Gewinner der digitalen Wirtschaft. Finanznachrichten 27 Jan 2016. www.accenture.com/technologyvision\#techvision2016. Accessed 28 Mar 2016

Chambers J, Kirkland R (2016) Interview: Cisco's John Chambers on the digital era. http://www.mckinsey.com/industries/high-tech/our-insights/ciscos-john-chambers-on-thedigital-era. Accessed 28 Mar 2016

Combe M (2014a) Change Agility: Readiness for Strategy Implementation. Project Management Institute. https:// www.pmi.org/ /media/PDF/learning/change-agility-readiness-for-strategy-implementation.ashx. Accessed 28 Mar 2016

Combe M (2014b) Change Readiness: Focusing Change Management Where It Counts. Project Management Institute. https://www.pmi.org/learning/ /media/PDF/Knowledge\%20Center/Focusing-Change-Management-Where-it-Counts.ashx. Accessed 28 Mar 2016

IBM (2008) Making change work: Continuing the enterprise of the future conversation. http://www-935.ibm.com/services/us/en/it-services/gts-it-service-home-page-1.html. Accessed 28 Mar 2016

Ringshausen H (2000) Die Bedeutung von Organisationstheorien für die betriebliche Weiterbildung. Eine theoriekritische Diskussion unter besonderer Berücksichtigung transdisziplinärer Ansätze. Hampp, München, Mering. ISBN: 978-387988-474-2

Schaubroeck R, Tarczewski FH, Theunissen R (2016) Making collaboration across functions a reality. Fast-changing global markets put a premium on simplifying processes radically and breaking through silos. http://www.mckinsey.com/ business-functions/organization/our-insights/making-collaboration-across-functions-a-reality. Accessed 28 Mar 2016

Trompenaars F, Hampden-Turner C (2012) Riding the Waves of Culture. Understanding Cultural Diversity in Business, 3rd edn. Brealey, London. ISBN: 978-1-904838-38-8

Autor

Prof. Dr. Hagen Ringshausen

Technische Hochschule Wildau/

DRC Consulting GmbH, Kassel

ringshausen@drc-consulting.de

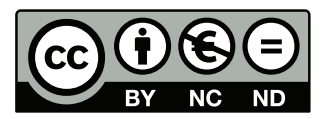

Dieser Beitrag ist unter der

Creative-Commons-Lizenz CC BY-NC-ND lizensiert. 